(From the Herzstein Research Laboratory of the University of California Berkeley, Cal.)

\title{
Ueber die allgemeinen Methoden der künstlichen Parthenogenese.
}

Von

\section{Jaeques Loeb.}

\section{Versuche an Annelideiern.}

1. Nachdem ich mich überzeugt hatte, dass die rein osmotische Methode der Entwicklungserregung des Seeigeleies in Wirklichkeit eine Entwicklungserregung durch Basen ist, während die Erhöhung des osmotischen Druckes nur als secundärer Umstand in Betracht kommt ${ }^{1}$ ), musste die Frage aufgeworfen werden, ob dieser Befund nicht vielleicht für alle Fälle osmotischer Entwicklungserregung gilt. Das scheint in der That der Fall zu sein, wie Versuche an Annelideneiern (Polynoë) und Molluskeneiern (Lottia gigantea) ergeben haben.

Die Eier von Polynoë sind im Allgemeinen unreif. In normalem Seewasser reifen sie nur, wenn sie befruchtet werden. Sie bilden alsdann eine Membran, werfen die beiden Polkörperchen aus und furchen sich. Bei $15^{\circ} \mathrm{C}$. erreichen solche Eier in etwa 8 Stunden das Larvenstadium und fangen an zu schwimmen. Werden die Eier nicht befruchtet, so bilden sie in normalem Seewasser keine Membran, stossen die Polkörperchen nicht aus und zerfallen in etwa 24 Stunden. Bringt man dagegen die unbefruchteten Eier von Polynoë in $50 \mathrm{ccm}$ Seewasser $+1,5 \mathrm{ccm} \mathrm{n/10} \mathrm{NaHO,} \mathrm{so} \mathrm{bilden} \mathrm{alle} \mathrm{Fier} \mathrm{im} \mathrm{Laufe} \mathrm{von}$ etwa $2-3$ Stunden (bei $15^{\circ}$ C.) Membranen, stossen beide Polkörperchen aus, und einige fangen an, sich völlig regelmässig zu furchen. (Die Furchung verläuft aber viel langsamer als bei den befruchteten Eiern.) Diese Eier und wohl auch andere, bei denen man keine Furchung wahrnimmt, wandeln sich in Larven um, die aber erst sehr spät, gewöhnlich etwa nach $30-40$ Stunden, anfangen

1) Pflüger's Arch. Bd. 118 S. 36. 1907. 
zu schwimmen. Dieser Versuch, den ich oft wiederholt habe, beweist also, dass, wenn die unbefruchteten Eier von Polynoë in hyperalkalisches Seewasser gebracht werden und dauernd in demselben bleiben, ein Theil derselben sich zu schwimmenden Larven entwickelt.

Eine wesentliche Bedingung für diese Entwicklung ist ausgiebige Sauerstoffversorgung. Wenn man nämlich die Eier in flache Uhrsehälchen bringt, die mit einer Glasplatte lose bedeckt sind, so entwickeln sich relativ viel mehr Eier, als wenn man sie in einer Schale hält, in der sie mit einer $1^{1 / 2}-2 \mathrm{~cm}$ hohen Wasserschicht bedeckt sind. Hemmt man die Oxydationsprocesse im Ei durch Zusatz von $1-2 \mathrm{cem}$ einer $1 / 10 \%$ igen KCN-Lösung zu $50 \mathrm{ccm}$ des hyperalkalischen Seewassers, so findet weder eine Reifung noch eine Entwicklung der Eier statt. Sobald aber alles CN durch Bildung von HCN und nachherige Verdunstung aus der Lösung versehwunden ist, reifen und entwickeln sich die Eier.

Fügt man $1 \mathrm{ccm} n / 10 \mathrm{NaHO}$ zu $50 \mathrm{ccm}$ Seewasser, so reifen die Eier ebenfalls, und einige können sich entwickeln. Bei Zusatz von $0,5 \mathrm{cem} \mathrm{n} / 10 \mathrm{NaHO}$ zu $50 \mathrm{ccm}$ Seewasser trat aber meist keine Reifung und niemals eine Entwicklung ein.

2. Ich hatte nun schon vor dem Anstellen dieser Versuche gefunden, dass man auch auf osmotischem Wege die Eier von Polynoë zur Entwicklung bringen kann, nämlich wenn man sie etwa 2 Stunden in eine Mischung von $50 \mathrm{ccm}$ Seewasser $+10 \mathrm{ccm} \mathrm{21/2}$ norm. NaCl bringt. Da nun Seewasser schwach alkalisch ist, so lag es auf Grund der Erfahrungen am Seeigelei nahe, zu vermuthen, dass es sich hier um die Combination von zwei Agentien handele, nämlich einer Wirkung von Hydroxylionen und einer Wirkung der Erhöhung des osmotischen Druckes. Das ist in der That der Fall. Ersetzt man das Seewasser durch eine neutrale van't Hoff'sche Lösung, so kann man durch eine Erhöhung des osmotischen Druckes einer solchen Lösung die unbefruchteten Eier von Polynoë nicht zur Entwicklung erregen, während das sehr gut gelingt, wenn man der Lösung eine kleine Menge Natronlauge zusetzt. In $50 \mathrm{ccm}$ der neutralen van't Hoff'schen Lösung wurden 0 resp. $0,5 \mathrm{ccm}$ n/10 NaHO zugefügt. Die Eier blieben 2 Stunden in diesen Lösungen und wurden dann in normales Seewasser übertragen. Die Eier, die in der neutralen hypertonischen Lösung gewesen waren, blieben meist unreif und keines furchte sich oder entwickelte sich zur Larve. Die Eier, die in der alkalischen hypertonischen Lösung gewesen waren, 
furchten sich zum Theil und entwickelten sich in etwa 24 Stunden zu schwimmenden Larven.

Ich habe Eier von Polynoë länger als 2 Stunden, nämlich 3-6 Stunden lang, in neutraler bypertonischer van't $\mathrm{H}$ off'scher Lösung gehalten, ohne dass sie sich entwickelten, wenn man sie in normales Seewasser zurückbrachte. Brachte man sie aber in byperalkalisches Seewasser, so entwickelten sie sich.

3. Es ist nun von Bedentung, dass auch beim Ei von Polynoë die entwicklungserregende Wirkung von Basen erhöht wird, wenn man die Eier ausserdem noch mit hypertonischem Seewasser behandelt. Ein Beispiel mag das erläutern. Die unbefruchteten Eier eines Weibchens von Polynoë wurden in $50 \mathrm{ccm}$ Seewasser $+1,5 \mathrm{ccm}$ n/10 NaHO gebracht. Ein Theil der Eier blieb dauernd in dieser Lösung, der Rest wurde nach 4 Stunden (als die Polkörperchen ausgeworfen waren $)^{1}$ ) in $50 \mathrm{ccm}$ Seewasser $+9 \mathrm{~cm} 2^{1 / 2}$ norm. $\mathrm{NaCl}$ gebracht und von hier 2 Stunden später in normales Seewasser zurückgebracht. 4 Stunden später waren $1 / 2 \%$ der dauernd im hyperalkalischen Seewasser gebliebenen Eier im Zweizellstadium, während ca. $30 \%$ der Eier gefurcht waren, die ausserdem noch mit hypertonischem Seewasser behandelt waren. Die Furchung war auch hier bis zum Achtzellstadium fortgeschritten. Am nächsten Morgen, also 24 Stunden nach Beginn des Versuches, waren die mit hypertonischem Seewasser behandelten Eier praktisch alle zu schwimmenden Larven entwickelt. Bei den im hyperalkalischen Seewasser befindlichen Larven stellten sich die Schwimmbewegungen erst 6-24 Stunden später ein, und die Zahl der sich entwickelnden Larven war erheblich kleiner. Derselbe Versuch wurde von mir und Herrn Wolfsohn sehr oft mit demselben Erfolg wiederholt.

Lässt man die Eier nur so lange in hyperalkalischem Seewasser, bis sie reif sind (d. h. eine Membran gebildet und die beiden Polkörperchen ausgestossen haben), und bringt man sie dann in normales Seewasser zurück, so entwickeln sie sich meist nicht. Man muss sie bei $15^{\circ}$ C. mindestens $5-6$ Stunden im hyperalkalischen Seewasser lassen, ehe sie im Stande sind, sich in normalem Seewasser weiter

1) Ich möchte für Morphologen bemerken, dass in all diesen Versuchen ausnahmslos zwei Polkörperchen von jedem unbefruchteten Ei abgegeben wurden, und dass also die Hypothese von der befruchtenden Wirkung des zweiten Polkörperchens sicher falsch ist. 
zu entwickeln. Aber es bilden sich mehr Larven, wenn die Eier nicht in normales Seewasser übertragen werden, sondern in der hyperalkalischen Lösung bleiben, und die Resultate sind noch viel besser, wenn man die Eier ausser mit hyperalkalischem auch noch mit hypertonischem Seewasser behandelt. Es ist also klar, dass bei Polynoë die künstliche Partbenogenese durch Basen veranlasst wird, während die Erhöhung des osmotischen Druckes nur zur Verbesserung der durch die Wirkung der Basen eingeleiteten Entwicklung beiträgt. Bei allen diesen Versuchen erwies sich die Sauerstoffversorgung der Eier als eine wesentliche, unerlässliche Bedingung. Alle Versuche, die Eier von Polynoë durch Säuren zur Entwicklung zu bringen, sind bisher gescheitert.

\section{Versuche an Molluskeneiern.}

Als Beispiel aus der Gruppe der Mollusken diente Lottia gigantea. Ich habe schon früher mitgetheilt, dass in allen meinen Beobachtungen die Eier dieser Form unreif waren, und dass sie in normalem Seewasser niemals reifen. Es ist völlig unmöglich, derartige Eier durch den Samen der eigenen Art zu befruchten. Sie reifen jedoch, wenn man sie einige Stunden in hyperalkalisches Seewasser bringt (50 ccm Seewasser $+1,0$ oder $1,5 \mathrm{ccm} n / 10 \mathrm{NaHO}$ ), vorausgesetzt, dass freier Sauerstoff in hinreichender Menge den Eiern zur Verfügung steht. Man kann sie ebenfalls zur Reife bringen, wenn man sie einige Stunden in gewöhnliches Seewasser bringt, dessen Concentration man erhöht hat. In letzterem Falle reifen nur wenige Eier, aber dieselben verharren nicht im Reifezustand, sondern entwickeln sich parthenogenetisch zu Larven. Auch hierbei ist die Gegenwart von freiem Sauerstoff unerlässlich. ${ }^{1}$ )

Ich führte nun neuerdings eine Reihe von Versuchen aus, um festzustellen, inwieweit die Concentration der Hydroxylionen für die osmoticche Entwicklungserregung der unbefruchteten Eier von Lottia in Betracht kommt. Es zeigte sich, dass diese Variable auch hier von ausschlaggebender Bedeutung ist. Es war selbstverständlich, dass hier als Ausgangslösung nicht Seewasser, sondern eine neutrale van't $\mathrm{H}$ off'sche Lösung ( $\left.\mathrm{NaCl}, \mathrm{KCl}, \mathrm{CaCl}_{2}\right)$ benutzt wurde. In einem Versuche wurde zu einer Mischung von $50 \mathrm{ccm}$ dieser Lösung + $10 \mathrm{cem} \mathrm{21/2}$ norm. NaCl H $0,0,1,0,2,0,5,1,0,2,0^{n / 10} \mathrm{NaO} \mathrm{zu}-$

1) Loeb, Untersuchungen über künstliche Parthenogenese. Leipzig 1906. 
gefügt. Die Eier von einem Weibchen wurden in diese hypertonische Lösung vertheilt und in bestimmten Intervallen von 30 Minuten bis 5 Stunden in normales Seewasser übertragen. Die Temperatur war $18^{\circ} \mathrm{C}$. Von den Eiern, die in der neutralen hypertonischen Lösung waren, reiften nur sehr wenige, nachdem sie mehr als 2 Stunden der hypertonischen Lösung ausgesetzt gewesen waren, und kein Ei entwickelte sich. Der Zusatz von $0,1 \mathrm{~cm} n / 10 \mathrm{NaHO}$ verbesserte die Wirkung nur wenig. Es reiften etwa $2 \%$ der Eier, die 150 Minuten in dieser Lösung gewesen waren, und ich fand auch später eine schwimmende Larve unter diesen. Die früher oder später aus der Lösung genommenen Eier gaben schlechtere Resultate. Der Zusatz von $0,2 \mathrm{ccm}$ n/10 $\mathrm{NaHO} z \mathrm{zu} 60 \mathrm{ccm}$ der hypertonischen Lösung ergab schon bessere Resultate. Schon unter den nach einer Stunde herausgenommenen Eiern reiften $2 \%$, aus den nach 90 Minuten herausgenommenen reiften $5 \%$, aus den nach 2 Stunden herausgenommenen waren $10 \%$ reif u. s. f. Eine kleine Zahl dieser Eier entwickelte sich auch zu schwimmenden Larven. Unvergleichlich besser waren die Resultate bei Zusatz von 0,5 und $1,0 \mathrm{ccm}$ NaHO. Die nach 2 Stunden aus dieser Lösung in normales Seewasser übertragenen Eier entwickelten sich praktisch alle zu schwimmenden Larven; der Zusatz von $0,5 \mathrm{ccm} \mathrm{n} / 10 \mathrm{NaHO}$ war günstiger als der Zusatz von 1,0 ccm NaHO, da die letztere Quantität sich bereits als schädlich erwies. Die Lösung, zu der $2,0 \mathrm{cem}$ NaHO zugefügt wurde, gab schlechtere Resultate, da die Eier offenbar infolge der hohen Concentration der Hydroxylionen litten.

Dieser Versuch, der mehrfach wiederholt und modificirt wurde und stets dasselbe Resultat gab, zeigt, dass auch bei der osmotischen Entwicklungserregung der Molluskeneier die Concentration der Hydroxylionen eine wichtige Variable ist.

Aber ein Umstand bei den Versuchen über die Reifung war sehr merkwürdig. Während die Eier von Lottia in normalem Seewasser nie reiften, reiften sie, wie erwähnt, wenn man den osmotischen Druck des Seewassers erhöhte, oder wenn man die Concentration der Hydroxylionen durch Zusatz von Basen erhöhte. Da die physiologische Wirkung der Erhöhung des osmotischen Druckes auf das $\mathrm{Ei}$ noch in vielen Punkten ein Räthsel ist, so drängte sich mir der Gedanke auf, ob nicht am Ende die Erhöhung des osmotischen Druckes des Seewassers zum Theil wenigstens dieselben chemischen Wirkungen im Ei hervorbringt wie der Zusatz von Natronlauge; sei es, 
dass die Concentration der Hydroxylionen im Ei erhöht wird, sei es, dass ähnliche chemische Umsetzungen im Ei infolge der Wasserentziehung hervorgebracht werden wie bei Erhöhung der Concentration der Hydroxylionen. Ich prüfte diesen Gedanken, indem ich die unbefruchteten Eier von Lottia in hypertonische neutrale Lösungen von verschiedenem osmotischen Druck brachte. Es zeigte sich in der That, dass bei höherem osmotischen Druck in einer neutralen hypertonischen van't Hoff'schen Lösung mehr Eier reiften als bei geringerem osmotischen Druck, obwohl in beiden Fällen die Zahl der reifenden Eier ganz erheblich geringer war, als bei Zusatz von Natronlauge. So reiften nahezu doppelt so viele Fier in derselben Zeit bei Zusatz von $17 \mathrm{ccm} 2^{1 / 2}$ norm. $\mathrm{NaCl}$ zu $50 \mathrm{ccm}$ der van't H off' schen Lösung als bei Zusatz von $9 \mathrm{ccm}$. Es ist möglich, dass es sich hier um ein Beispiel der neuerdings von Szyszkowski gefundenen Lösung der Dissociation von Alkalien und Säuren durch Zusatz von Neutralsalzen handelt ${ }^{\mathbf{1}}$ ).

\section{Versuche am Seeigelei.}

1. Ich komme nun $\mathrm{zu}$ dem theoretisch wichtigsten Theil dieser Versuche, nämlich zu dem Nachweis, dass bei der künstlichen Parthenogenese Säuren und Basen gleich oder ähnlich wirken, obwohl bei manchen Formen, wie Polynoë und Lottia, soweit wir bis jetzt wissen, anscheinend nur Alkalien, bei anderen anscheinend nur Säuren zur Anwendung kommen können. Das letztere mag aber bei weiteren Versuchen sich als eine zu enge Auffassung erweisen, und es mag sich herausstellen, dass überall Säuren und Alkalien in gleichem oder ähnlichem Sinne wirken. Für das Seeigelei ist dás sicher der Fall, wie wir jetzt zeigen können.

Ich habe in früheren Arbeiten gezeigt, dass man durch Säure die Entwicklung von Seeigeleiern hervorrufen kann, vorausgesetzt, dass mit der Säurebehandlung auch eine Behandlung mit einer hypertonischen Lösung combinirt wird. Die Säurewirkung besteht in der Hervorrufung des Membranbildungsprocesses von Seiten des Eies. Dabei macht es einen grossen Unterschied, ob die Säurebehandlung der Behandlung des Eies mit der hypertonischen Lösung voraufgeht oder ihr folgt. Ruft man zuerst die Membranbildung durch eine Säure hervor, so braucht man die Eier hinterher nur

1) Zeitschr. f. physikal. Chemie Bd. 58 S. 420. 1907. 
etwa $1 / 2-1$ Stunde dem hypertonischen Seewasser auszusetzen; bei der umgekehrten Reihenfolge müssen die Eier $1 \frac{1 / 2}{\text { bis }}$ 2 Stunden der hypertonischen Lösung ausgesetzt werden, um sich entwickeln zu können, und auch dann ist die Entwicklung nicht so schön wie bei der umgekehrten Reihenfolge.

Ganz genau so verhält es sich, wenn man statt der Säure Basen anwendet. Ich habe in einer früheren Abhandlung berichtet, dass man die unbefruchteten Seeigeleier zur Entwicklung veranlassen kann, wenn man sie erst 2 Stunden einer neutralen hypertonischen van't Hoff'schen Lösung ( $50 \mathrm{ccm}$ m/2 van't $\mathrm{H}$ off'schen Lösung $+8 \mathrm{~cm} 2^{1 / 2} \mathrm{n} \mathrm{NaCl}$ ) aussetzt und sie hinterher $11 / 2$ bis 2 Stunden lang in hyperalkalisches Seewasser bringt $(50 \mathrm{ccm}$ Seewasser $+1,5 \mathrm{ccm} n / 10 \mathrm{NaHO}$ ) bei einer Temperatur von ungefähr $15^{\circ} \mathrm{C}$. Ich habe nun neuerdings Versuche angestellt, bei denen die Eier zuerst in die byperalkalische und dann in die hypertonische Lösung gebracht wurden, und gefunden, dass in diesem Falle die Expositionsdauer in der hypertonischen Lösung viel geringer ist, nämlich bloss $1 / 2$ bis 1 Stunde. Ein Beispiel möge das erläutern. Zu je $50 \mathrm{ccm}$ einer mit Seewasser isotonischen (halbgrammolecularen) van't $\mathrm{H}$ off'sehen Lösung $\left(\mathrm{NaCl}, \mathrm{KCl}, \mathrm{CaCl}_{2}\right.$ ) wurde $0,0,5,1,0,1,5$ und $2,0 \mathrm{ccm}{ }^{n} / \mathbf{1} 0$ NaHO zugesetzt. Die Eier eines Weibchens wurden in dieser Lösung vertheilt und 175 Minuten in derselben gelassen. Dann wurden sie in neutrale hypertonische Lösungen, nämlich $50 \mathrm{ccm}$ van't Hoff'sche Lösung $+10 \mathrm{ccm}$ $2^{1 / 2}$ norm. $\mathrm{NaCl}$ gebracht und von hier nach 35 und 50 Minuten in normales Seewasser übertragen. Die Temperatur war $14^{\circ} \mathrm{C}$. Die Eier, welche in der neutralen van't $\mathrm{H}_{0}$ ff'schen Lösung gewesen waren, furchten sich weder, noch entwickelten sie sich. Eine Portion dieser Eier blieb $2^{3 / 4}$ Stunden in der neutralen hypertonischen Lösung, ohne dass eine Spur einer Entwicklung eintrat. Bei den Eiern dagegen, welche nach der Behandlung mit der alkalischen $\mathrm{van}$ 't $\mathrm{H}$ off'schen Lösung 35 resp. 50 Minuten in die hypertonische Lösung gebracht wurden, trat eine Entwicklung zu Larven ein, aber nur bei solchen Eiern, die in Folge der Behandlung mit alkalischen Lösungen eine Membran bildeten. Der Zusatz von 0,5 und $1,0 \mathrm{ccm} \mathrm{NaHO}$ gab gute Resultate, 1,5 und $2,0 \mathrm{ccm} \mathrm{NaHO}$ schädigte die Eier; vielleicht würden auch diese Lösungen gute Resultate gegeben haben, wenn die Eier nicht so lange darin verweilt hätten. Die Eier, welche bei dieser Versuchsmethode eine Membran bildeten, 
furchten sich auch meist in normaler Weise. Die Membranbildung fand nicht statt, während die Eier in der alkalischen Lösung waren, sondern anscheinend erst während oder nach der Behandlung mit der hypertonischen Lösung. Die Membran lag auch meist dem $\mathrm{Ei}$ viel dichter an als die Säuremembran. In anderen Versuchsreihen wurde festgestellt, dass die mit den erwähnten hyperalkalischen Lösungen 2 Stunden lang behandelten Eier nur dann sich zu normalen Larven entwickelten, wenn sie nicht länger als 1 Stunde in der hypertonischen Lösung blieben. Ein längerer Aufenthalt tötete die Eier.

Wir sehen also, dass die Entwicklungserregung durch Basen der durch Säuren durchaus vergleichbar ist, nur dass die Wirkung der Basen sehr viel langsamer ist als die der Säuren.

2. Wir dürfen nun die Frage aufwerfen, wie es kommt, dass das Ei nur kurze Zeit in der hypertonischen Lösung zu bleiben braucht, wenn die Behandlung mit Alkali oder Säure voraufgeht, während es drei- bis viermal so lange in der hypertonischen Lösung bleiben muss, wenn die Behandlung mit Säure oder Alkali der Behandlung des Eies mit der hypertonischen Lösung nachfolgt.

Ich habe bereits früher gezeigt, dass die Hervorrufung der Membranbildung genügt, um die der Entwicklung zu Grunde liegenden chemischen Processe im Ei, in erster Linie Oxydationsprocesse, einzuleiten, dass diese Oxydationsprocesse aber in falschen Bahnen verlaufen und zum Tode des Eies führten. Die Wirkung der hypertonischen Lösung ist eine rein chemische, was durch die Bestimmungen des Temperaturcoëfficienten (der $\geqq 3$ ist) bewiesen wird, sowie durch den Umstand, dass die hypertonische Lösung nur in Gegenwart von freiem Sauerstoff wirksam ist ${ }^{1}$ ). Ich stelle mir vor, dass im Ei, während es in der hypertonischen Lösung ist, Stoffe entstehen, welche die Oxydationsvorgänge wieder in die richtigen Bahnen lenken. Die Bildung dieser Stoffe erfolgt nun viel rascher, wenn im Ei bereits in Folge der Membranbildung die Entwicklung angeregt ist, als wenn das ruhende $\mathrm{Ei}$ in die hypertonische Lösung gebracht wird.

\section{Theoretische Bemerkungen.}

Es scheint mir, dass durch diese Versuche die Methodik der künstlichen Parthenogenese eine wesentliche Vereinfachung erfährt.

1) Lu o b, Biochem. Zeitschr. Bd. I S. 183. 1906. 
Es sind im Wesentlichen zwei Methoden, die zur Anwendung gelangen, Behandlung der Eier mit Säuren oder mit Basen. Für die Eier mancher Formen scheinen nur die Basen, bei anderen Formen nur die Säuren und in wieder anderen Formen beide wirksam zu sein. Bei gewissen Eiern ist die Behandlung mit diesen Stoffen ausreichend, bei anderen muss noch eine Nachbehandlung mit hypertonischen Lösungen erfolgen.

Fragen wir nun, was diese beiden Agentien, Säuren und Basen, im Ei verändern, so müssen wir im Sinne behalten, dass Oxydationsprocesse die Voraussetzung und Grundlage aller Entwicklungsvorgänge im Ei sind, und ich habe schon in voraufgehenden Arbeiten gezeigt, dass zweifellos diese Oxydationsprocesse im $\mathrm{Ei}$ durch die Säure- und Alkalibehandlung sowohl wie durch das Eindringen des Spermatozoons in Gang gesetzt oder beschleunigt werden ${ }^{1}$ ). Aber diese Anregung von Oxydationsprocessen ist offenbar keine directe, sondern eine indirecte Wirkung der Säuren und Basen; die directe Wirkung ist die Hervorrufung eines chemischen Vorganges, der meist als meehanische Folge die Abhebung der äusseren Lamelle des Eies, die sogenannte Membranbildung zur Folge hat. Das folgt erstens daraus, dass, wenn bei der Säure- und Alkalibehandlung nur ein Theil der Eier Membranen bildet, uur diejenigen Eier sich entwickeln, welche eine Membran besitzen; es folgt zweitens daraus, dass auch andere Methoden, durch welche eine Membranbildung bewirkt wird, z. B. die Behandlung der Eier mit fettlösenden Stoffen, wie Benzol oder Amylen, oder mit dem Blutserum von Sipunculiden, die Entwicklung anregen.

Wir werden so auf die Frage geführt, wie wir uns den $\mathrm{Zu}$ sammenhang des Membranbildungsprocesses mit der Anregung von Oxydationsprocessen vorstellen können. Ich habe nun schon früher auf den Zusammenhang zwischen Cytolyse des Eies und der Membranbildung hingewiesen, und bei allen neueren Versuchen hat sich dieser Zusammenhang immer wieder gezeigt. Bei der Behandlung der Eier mit Benzol oder Amylen folgt der Membranbildung die Cytolyse fast unmittelbar, und in einer neueren Arbeit babe ich auch mitgetheilt, dass selbst die zur Entwicklung des Seeigeleies optimale Concentration der Hydroxylionen das $\mathrm{Ei}$ rasch cytolysiren würde, wenn nicht das Calcium des Seewassers die Cytolyse hinderte ${ }^{2}$ ).

1) 1. c.

2) Loeb, Biochem. Zeitschr. Bd. 2 S. 81. 1906. 
Der Membranbildungsprocess im Ei ist ein Durchgangsstadium im Process der Cytolyse oder Schattenbildung. Wenn wir uns nun nach einer Erklärung dieses Vorgangs umsehen, so scheinen die eben veröffentlichten Ansichten von L. Liebermann ${ }^{1}$ ) besondere Beachtung $\mathrm{zu}$ verdienen. Lieberman nimmt im Anschluss an Hoppe-Seyler an, dass in den rothen Blutkörperchen der Farbstoff mit dem Stroma chemisch verbunden ist, und dass bei der Hämolyse durch Säure oder Seifen diese Verbindung gespalten wird, wodurch das Hämoglobin frei wird und eventuell in die umgebende Flüssigkeit entweichen kaǹn. In Analogie damit könnte man sich vorstellen, dass auch im $\mathrm{Ei}$ der Act der Befruchtung durch den Samen oder durch Säuren und Alkalien oder fettlösliche Stoffe in chemischen Spaltungsvorgängen besteht. Als eine nebensächliche Wirkung dieser Spaltungsvorgänge tritt die Membranbildung ein. Wesentlich ist aber, dass durch diese Spaltungen die Oxydationen im Ei eintreten, die aller weiteren Entwicklung (der Nucleïnsynthese, ferner der Astrosphärenbildung und Kern- und Zelltheilung) zu Grunde liegen; sei es, dass ein Produkt dieser Spaltungsvorgänge Oxydasen oder deren Vorstufen sind, sei es, dass durch diese Spaltung erst das oxydirbare Material geliefert wird.

Selbst wenn wir von der Bedeutung des Membranbildungsprocesses ganz absehen wollten - wozu wir aber kein Recht haben so wird die Thatsache, dass Säuren sowohl wie Alkalien die künstliche Parthenogenese hervorrufen, die Chemiker an die Saponification von Estern erinnern; und in der That hat mir Herr Professor A r r henius, als ich ihm meine neueren Versuche mittheilte, diesen Gedanken auch brieflich ausgedrückt. Im Hinblick auf die Erfahrungen bei der Keimung ölbaltiger Samen hat dieser Gedanke etwas sehr Bestechendes. Wie die Beobachtungen von Pelouze und von $\mathrm{Niclo \textrm {x } ^ { 2 }}$ ) zejgen, liegt der Keimung hier eine Saponification von Estern zu Grunde. Für diese Saponification ist ein Enzym vorhanden, das aber nur in der Gegenwart einer Säure seine Wirksamkeit beginnen kann. Diese Säure wird vom Samen selbst geliefert, nämlich in der Form von Kohlensäure. Die Fettsäuren, welche durch die Saponification entstehen, liefern das Material für die Oxy-

1) Liebermann, Biochem. Zeitschr. Bd. 4 S. 25.1907.

2) Nicloux, Contribution à l'étude de la saponification des corps gras. Paris 1906. 
dation. Es liegt einstweilen aber kein Grund vor, diese Parallele zwischen dem Anfang des Keimungsprocesses und der Befruchtung zu stark zu betonen. Ich möchte aber nicht unterlassen, darauf hinzuweisen, dass ich schon vor 4 Jahren auf die Möglichkeit hinwies, dass bei den Eiern, welche "spontan" parthenogenetisch sind, die im $\mathrm{Ei}$ selbst gebildete Kohlensäure als das befruchtende Agens wirken dürfte ${ }^{1}$ ):

Zum Schluss möchte ich noch bemerken, dass Versuche an Chaetopterus und Amphitrite vermuthlich bei einer Wiederholung das hier Gesagte bestätigen werden. Für Amphitrite habe ich mich bereits früher davon überzeugt, dass bei genügender Sauerstoffversorgung der Alkaligehalt des Seewassers in Woods-Hole für die Anregung zur Entwicklung ausreicht und für Chaetopterus dürfte wohl das gleiche sich herausstellen. Bei der Anstellung meiner älteren Versuche war weder die Bedeutung der Concentration der Hydroxylionen noch die Bedeutung der Sauerstoffversorgung hinreichend gewürdigt worden, da erst die neueren Versuche die Bedeutung dieser Variabeln ans Licht gebracht haben.

1) Auf Grund meiner neueren Versuche könnten auch die Hydroxylionen der umgebenden Lösung, z. B. des Seewassers, diese Rolle übernehmen. 